\title{
Gasperini Syndrome as Clinical Manifestation of Pontine DEMYELINATION
}

\author{
M. Krasnianski ${ }^{1}$, T. Müller ${ }^{1}$, S. Zierz ${ }^{1}$, M. Winterholler ${ }^{2}$ \\ Departments of Neurology, \\ ${ }^{1}$ Martin-Luther-Universität Halle-Wittenberg, Halle (Saale) and ${ }^{2}$ Hospital Rummelsberg, Schwarzenbruck, Germany
}

\begin{abstract}
The Gasperini syndrome is a very rare brainstem disease characterized by the typical combination of ipsilateral lesions of the cranial nerves V-VII and dissociated contralateral hemihypesthesia, whereas both contralateral and ipsilateral hypacusis was described. Since the first description in 1912, only a few cases of this crossed brainstem syndrome were published so far. Pontine infarction and bleedings were the reported causes of the syndrome. Here we report a 44-year-old man with the classical Gasperini syndrome due to pontine demyelination in multiple sclerosis. The clinical findings were correlated with changes on MRI. The present case shows that classical crossed brainstem syndromes are topological terms not invariably associated with brainstem ischemia in particular vascular areas and may contribute to the differential diagnosis of peripheral facial nerve palsy.
\end{abstract}

Key words: Cranial nerves; crossed brainstem syndromes; facial nerve palsy; Gasperini syndrome

\section{INTRODUCTION}

The Italian physician Ubaldo Gasperini described in 1912 a patient with a lesion of the 5th, 6th and 7th cranial nerves of one side combined with a contralateral hypacusis and hemihypesthesia for pain and temperature, and only initially with a slight motor impairment [1]. The diagnosis was not confirmed by postmortem examination, but sudden onset of clinical symptoms was compatible with a vascular accident. Gasperini assumed a brainstem hemorrhage in the lateral caudal pontine tegmentum as the cause of the symptoms [1]. Only a few cases of this crossed brainstem syndrome caused by pontine infarctions were published so far $[2-4,6]$.

Here we report a patient with the classical Gasperini syndrome due to demyelination in multiple sclerosis. The clinical findings were correlated with changes on MRI.

\section{CASE REPORT}

A 44-year-old man was referred with the left-sided facial weakness and numbness of his right arm and leg. On admission, he was alert and oriented. He had an impaired abduction of the left eye with diplopia, a peripheral facial palsy with minimal lagophthalmus, a fa- cial hypesthesia, and a hypacusis on the left side. Speech, soft palate movements, swallowing, gag reflex, and tongue movements were normal. There was no disturbance of trapezius and sternocleidomastoid muscles, and no Horner's syndrome. The patient had no paresis. The deep tendon reflexes were symmetric and normal. Plantar responses were flexor. He had no ataxia. The patient had a right hemihypesthesia for pain and temperature.

T2-weighted cranial MRI revealed a high signal intensity area in the left lateral caudal pons (Fig. 1) and several smaller demyelinating lesions in the periventricular white matter. CSF analysis yielded a slight lymphocytic pleocytosis (36 cells $/ \mathrm{mm}^{3}$; normal $<5$ cells $/ \mathrm{mm}^{3}$ ) and oligoclonal bands.

The patient was treated with intravenously administered high-dose cortisone for 5 days. All neurological signs and symptoms with the exception of the mild facial nerve paresis on the left side gradually disappeared within one week.

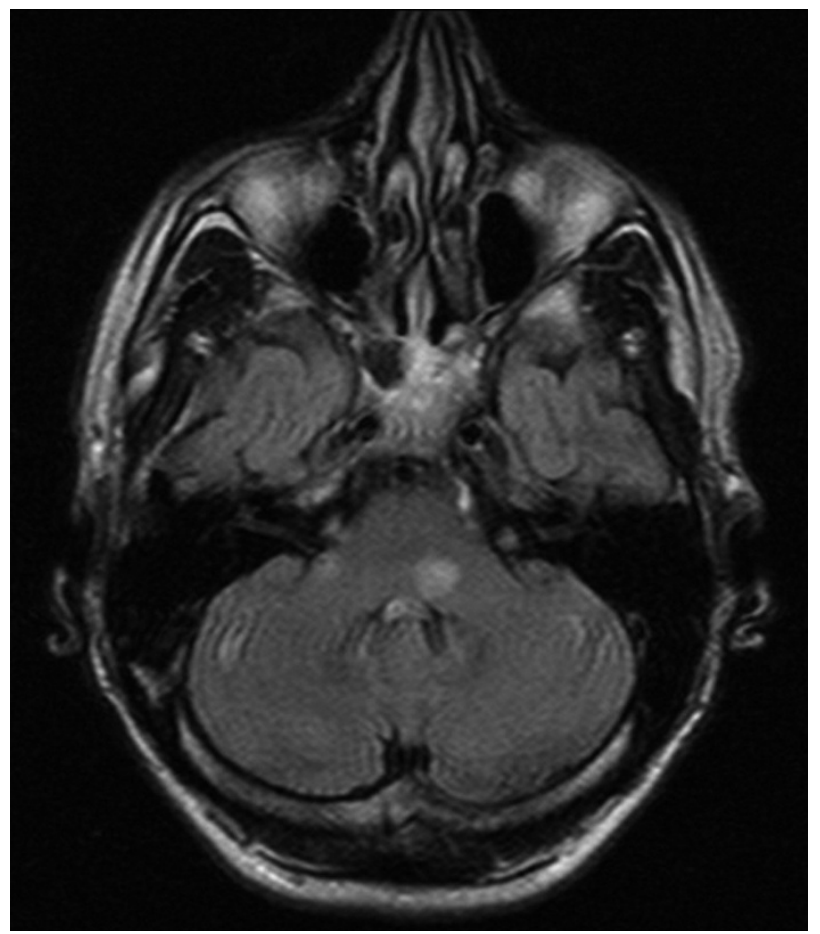

Fig. 1. FLAIR-weighted cranial MRI showing an area of increased signal intensity in the left-sided lateral caudal pontine tegmentum. 


\section{DISCUSSION}

The constellation of signs and symptoms in our patient corresponded to the Gasperini syndrome [1]. The MR imaging showed a demyelinating area in the left caudal pons (Fig. 1). The anatomical basis of the Gasperini syndrome can be considered as a lesion of the left-sided lateral caudal pontine tegmentum involving the ipsilateral facial nerve nucleus (peripheral facial palsy), the abducens fibres (abducens nerve palsy), the acoustic fibres (hypacusis), the spinal trigeminal tract (facial hypesthesia), and the spinothalamic tract (contralateral hemihypesthesia for pain and temperature) (Fig. 2) [5].

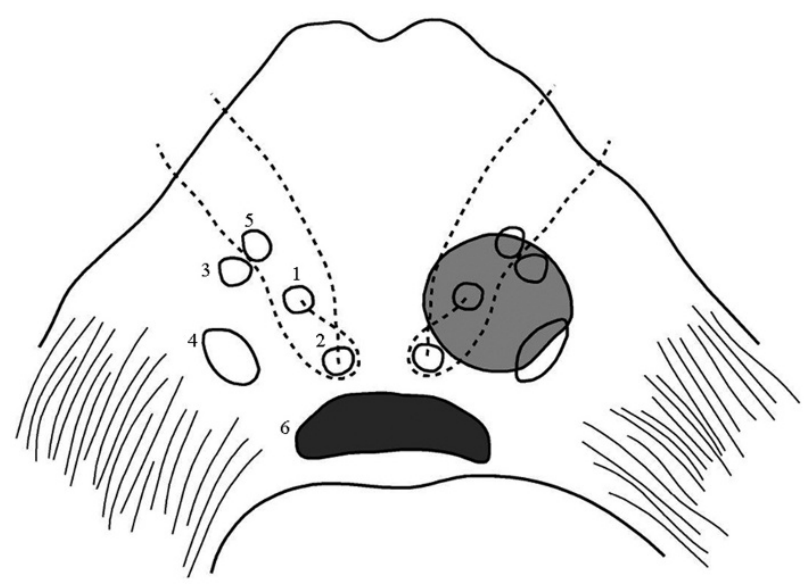

Fig. 2. Transverse section through the caudal pons showing: 1 - Facial nerve and nucleus; 2 - Abducent nerve and nucleus; 3 - Lateral lemniscus; 4 - Spinal trigeminal tract; 5 Spinothalamic tract; 6 - Fourth ventricle.

Since the lemniscus lateralis carries predominant information from the contralateral cochlea, affection of the lateral lemniscus usually results in predominant hypacusis of the contralateral side [2, 5]. However, affection of the lateral lemniscus did not cause a complete contralateral deafness or severe hypacusis described in the original case of Gasperini and in another recent case because of the binaural representation of the ascending auditory tracts $[1,6,7]$. Interestingly, Gasperini predicted the possibility of deafness on the ipsilateral side which was lately described in the majority of published cases of the Gasperini syndrome $[1,6]$. Presumably, the ipsilateral deafness or hypacusis may be caused by the involvement of the more peripheral portion of the acoustic pathway. In our patient the hypacusis was very slight and disappeared within 24 hours.
Since nearly 100 years only a very few cases of the Gasperini syndrome were published $[2-4,6]$. Pontine ischemia or bleeding due to venous angioma, vertebral artery occlusion, basilar artery stenosis , and small vessel disease associated with diabetes mellitus, hyperlipidemia or hypertension were reported as causes of the Gasperini syndrome so far [6]. Sometimes the Gasperini syndrome was even defined as the "AICA syndrome with abducens nerve palsy" [3].

The Gasperini syndrome with the typical combination of ipsilateral lesions of the cranial nerves V-VIII and dissociated contralateral hemihypesthesia in a patient with pontine demyelination shows that classical crossed brainstem syndromes are topological terms not invariably associated with brainstem ischemia in particular vascular areas. The present case may contribute to the differential diagnosis of peripheral facial nerve palsy, which also includes the Gasperini syndrome.

\section{REFERENCES}

1. Gasperini U. Di un caso di emorragia protuberenziale. Contributo clinico allo studio delle sindromi pontine inferiori. Rif Med 1912;28:880-883, 898-905.

2. Schwaninger M, Kanemoto K, Kölmel HW. Das Gasperini-Syndrom - eine Differentialdiagnose der peripheren Fazialisparese. Neurologische und bildgebende Befunde. HNO 1993;41:536-538.

3. Takamatsu K, Ohta T. A study of infarction in the region of the anterior inferior cerebellar artery. Rinsho Shinkeigaku 1995;35:621-625.

4. Roquer J, Lorenzo JL, Pou A. The anterior inferior cerebellar artery infarcts: a clinical-magnetic resonance imaging study. Acta Neurol Scand 1998;97:225-230.

5. Clara M. Anatomie des Nervensystems des Menschen. Leipzig: Johann Ambrosius Barth - Verlag, 1959:299-342.

6. Hayashi-Hayata M, Nakayasu H, Doi M, Fukada Y, Murakami T, Nakashima K. Gasperini syndrome, a report of two cases. Intern Med 2007;46:129-133

7. Brazis PW, Masdeu JC, Biller J. Localisation in clinical neurology. Fifth Edition. Philadelphia: Lippincott Williams \& Wilkins 2007: 307-324

Received: January 5, 2009 / Accepted: April 29, 2009

Address for correspondence:

PD Dr. med. Michael Krasnianski

Klinik für Neurologie

Martin-Luther-Universität Halle-Wittenberg

Ernst-Grube-Str. 40

06097 Halle (Saale)

Germany

Tel. $\quad+493455572858$

Fax: $\quad+493455572020$

E-mail: michael.krasnianski@medizin.uni-halle.de 